\section{Outreach teaching - the Leeds experience: reflections after one year}

\author{
H. L. Craddock ${ }^{1}$
}

IN BRIEF
- Outreach teaching can be an
important feature of undergraduate
dental education.
- Management of quality and consistency
in teaching is key to its success.
- Support for outreach teaching staff
is crucial.
- Additional learning outcomes beyond
the purely clinical can be achieved in
this setting.

Clinical experience in Outreach sites has taken place in many dental schools for a number of years. The wider recognition of Outreach as a useful learning environment has prompted many schools to share their experiences. The purpose of this paper is to share the findings of Leeds Dental Institute's experiences after the first year of Outreach learning in a purposebuilt Outreach clinic. The learning outcomes for this activity were carefully tailored to the experiences available within this community setting and built as an integrated part of the BChD dental curriculum. The successful introduction of a model of independent but well supported and managed clinical teaching units as part of Leeds Dental Institute has allowed our dental students to access both modern learning and teaching in a community based locality, while retaining access to the conventional centre of teaching and clinical excellence in a dental school and hospital.

\section{INTRODUCTION}

A number of dental schools who use Outreach teaching as part of their undergraduate curricula have shared their experiences of this type of learning environment in the dental literature.

This paper shares the Leeds experience across a range of themes including clinical facilities, management of quality standards, staffing, computerisation, teaching, evaluation, recording student activity, feedback and patient information.

Leeds Dental Institute came to undergraduate Outreach development a little later than some other schools. However, in doing so we have had a number of advantages, including benefiting from feedback from other schools' experiences, a longer lead-in time for detailed planning, and financial investment for increased student numbers. Many years of experience of clinical placement of dental care professional students in

Senior Lecturer/Honorary Consultant, Room 6129, Leeds Dental Institute, Clarendon Way, Leeds, LS2 9LU Correspondence to: Dr Helen Craddock Email:h.l.craddock@leeds.ac.uk

\section{Refereed Paper}

Accepted 1 March 2008

DOI: $10.1038 /$ bdj.2008.200

${ }^{\circ}$ British Dental Journal 2008; 204: 319-324 primary care trust community dental clinics was drawn on when planning the dedicated facilities. The developing Outreach programme has been carefully designed and monitored to supplement and enhance the delivery of longitudinal Learning Themes within the undergraduate curriculum.

As well as providing specific and unique learning opportunities for year 4 and 5 students, the incorporation of vertical themes within our new Integrated Curriculum means that these themes can be developed in a variety of Outreach settings.

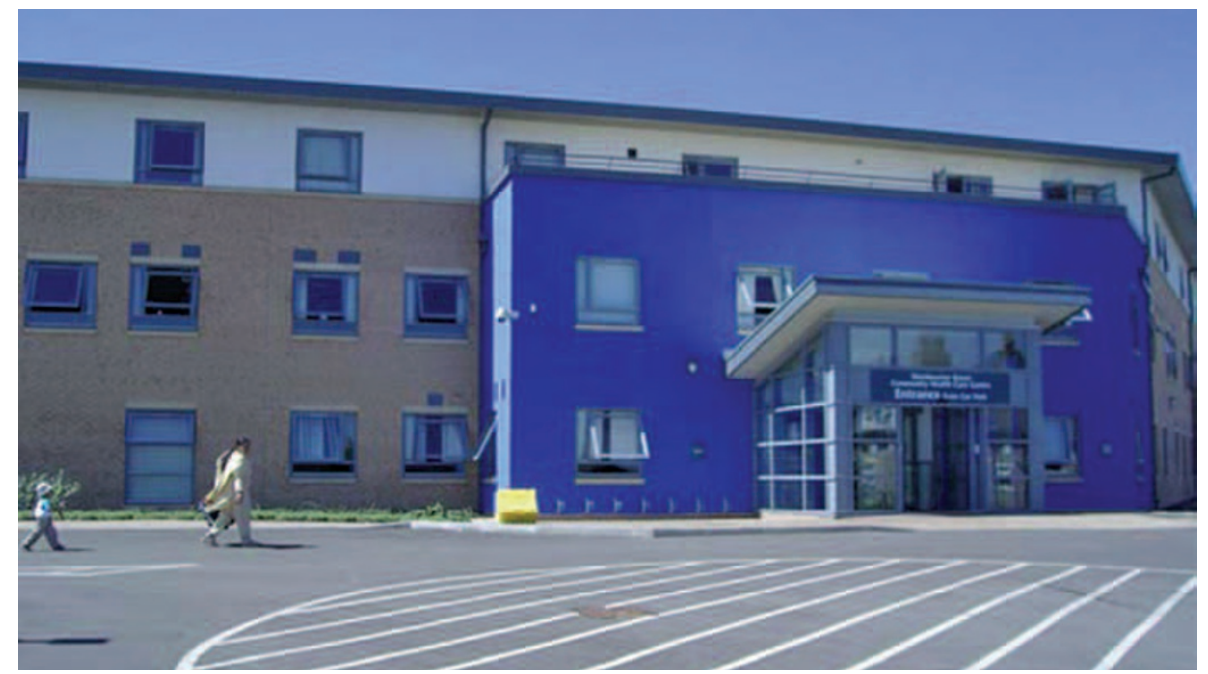

Fig. 1 Our first Outreach facility in Bradford

\section{Where we are now}

There are three 'designed for purpose' multi-disciplinary centres within our current Outreach portfolio within areas of high treatment need, the first of which has been operating for one year (Fig. 1) and the second will be functional within the next few months.

For our undergraduate students, many of our intended learning outcomes are specific to our Outreach Centre locations.

The intended learning outcomes for our overall Outreach experience include:

- Have experience of a wide range of cultural norms 
- Have practical experience of a range of skills and strategies for communicating with patients with language limitations

- Have experienced the use of translators for patient communication

- Have experience of whole patient care

- Have experience of treatment of family groups

- Be able to use electronic patient record systems

- Have experienced working in a team with all other groups of dental professionals to achieve high standards of patient care

- Have first hand experience of referring patients to other dental professionals

- Be able to carry out time management in the clinical setting

- Have experience of patient administration in a range of settings.

\section{Facilities}

The outreach clinic is a modern purpose built unit, which is designed and equipped to the highest standards. The equipment and materials are closely based on those provided at the Leeds Dental Institute. There are seven open plan cubicles within the facility (Fig. 2), similar in layout to that at the Dental Institute.

Digital radiography facilities linked to electronic patient management systems ensure that patient records are kept securely and are readily available.
A 'state of the art' decontamination centre is available to give students the opportunity of experiencing all stages of decontamination and sterilisation of dental instruments (Fig. 3).

\section{Clinical quality standards}

Clinical standards in Outreach are expected to be identical to those at Leeds Dental Institute and this is maintained via staff training, provision of nursing and administrative support and the use of our production laboratory within the Institute for all laboratory items.

\section{Inter-professional learning}

Leeds Dental Institute trains all members of the dental team. Inter-professional learning, which is already well established within our curricula, is reinforced in the Outreach setting, with dental undergraduates, student dental nurses, student hygiene therapists and student dental technicians all working within a cohesive team to achieve high standards of patient care via interdisciplinary co-operation and communication (Fig. 4). Tutors from dental nursing and hygiene therapy teach their respective team members whilst in the Outreach setting. This provides opportunities for tutors within each of these courses of study to interact and support each other's teaching practice.

\section{Staffing}

A dedicated teacher, who has had a formal four week induction period within the Institute, is employed and is supported and mentored by senior staff within the Dental Institute. The staff student ratio is one to eight, identical to that within the restorative undergraduate clinic. Chairside support is provided by student dental nurses, a senior dental nurse and there is full-time reception and clerical support available.

\section{Computerisation}

A fully integrated patient management system is in place, which allows students to record and store clinical data electronically, allows access to diaries and attendance records, and permits communication between reception and clinical areas. This system also allows retrieval and viewing of patient radiographs (Fig. 5).

\section{Teaching}

A clinical teacher with a background in general dental practice was recruited jointly between the Dental Institute and the Primary Care Trust. Her only role is the provision of high standards of clinical teaching. Leeds Dental Institute is responsible for this teacher's professional development and she has an individual mentor who is a full time member of the academic staff.

In addition to this a senior dental nurse was recruited for the Dental Outreach unit. The employment of both the clinical teacher and the senior dental

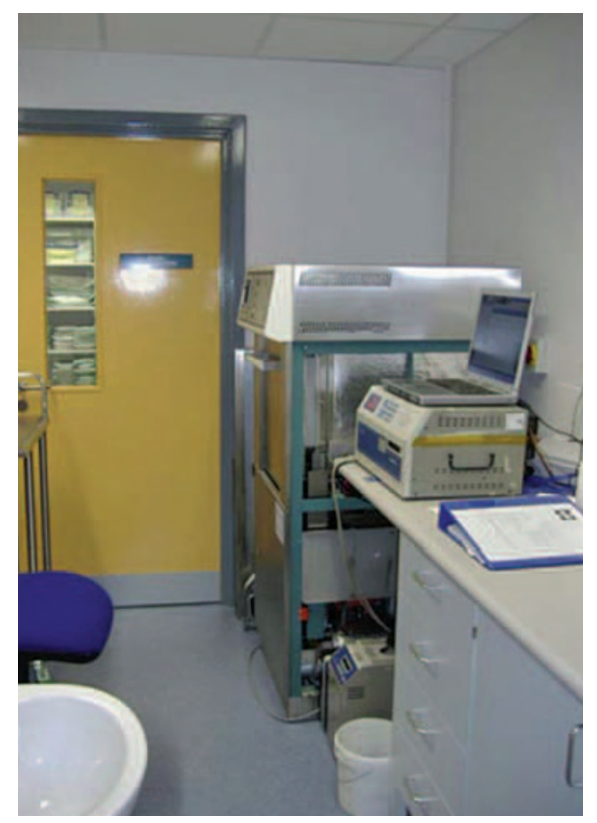

Fig. 3 Sterilisation section of three part decontamination unit 


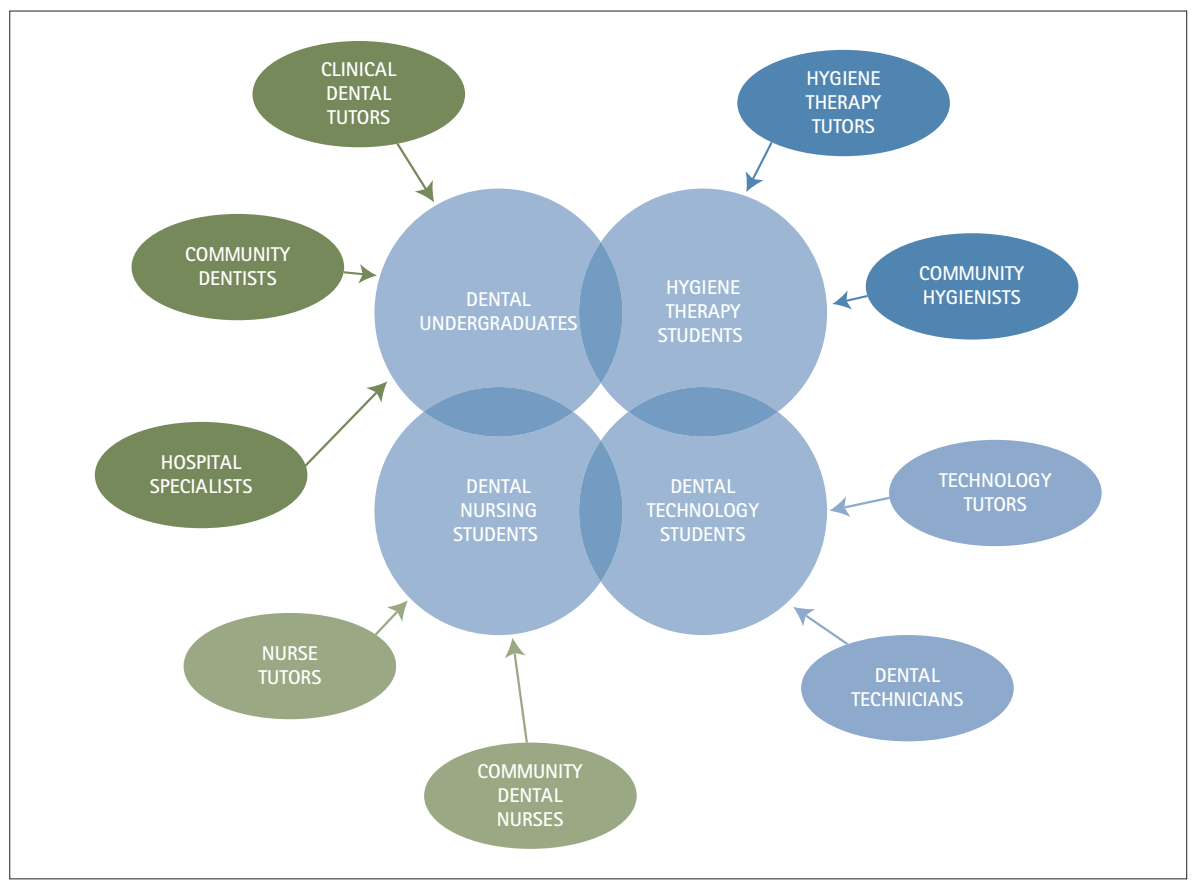

Fig. 4 Opportunities for interdisciplinary learning

nurse are supported by Dental Special Increment For Teaching (Dental SIFT) funding.

Opportunities are provided for the staff at all the Outreach sites (including our existing hygiene therapy sites) to meet for training and information exchange purposes, as well as mutual support and reflection around their roles. All our teachers are, after an initial familiarisation period, encouraged to attend courses at the university leading to the University of Leeds Teaching Award Level 2 and our first member of staff from Outreach will commence this in September 2007. This qualification is recognised by the Higher Education Council.

There are a number of training days per year when Outreach staff join staff employed at the Institute for various activities. Each member of Outreach staff also attends the Institute one day per week to carry out teaching and personal development activities, together with weekly meetings with their mentor.

\section{Evaluation}

Evaluation and feedback on student performance, both in terms of clinical work and patient management, is provided throughout clinical activity.

A marking scheme, marking criteria and proforma identical to that used in the Institute is used to assess and record student performance.

\section{Record of student activity}

Patients seen and treatments carried out are recorded electronically using the clinical management software systems. Activity reports are easily accessible to all students and supervisory staff.

\section{Feedback}

Student feedback is sought at the end of the one year placement. Questionnaires were sent to each student in the year and 48 completed questionnaires were received from 55 students attending the Outreach Clinic, a response rate of $87 \%$. A copy of the questionnaire is shown here (Appendix 1).

Outreach placement is seen as a complementary clinical experience, fulfilling learning outcomes that are less readily achieved in the dental hospital setting. In the questionnaire used, the egorised under five headings: strongly agree, agree, ambivalent, disagree and strongly disagree. The positive responses are indicated in blue, the negative in yellow.

$94 \%$ of students either strongly agreed or agreed with the statement: I better understand how culture can affect patient dental experience and attitude.

$83 \%$ of students either strongly agreed or agreed with the statement: I am aware of the skills necessary to communicate with patients for whom English is difficult. students' response to questions were cat-

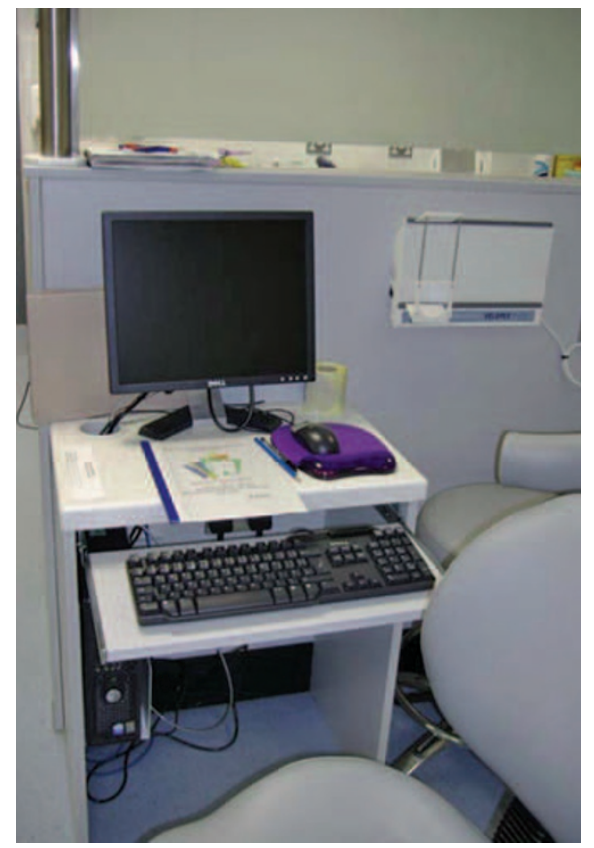

Fig. 5 Digital patient management and radiographic viewing facility

$73 \%$ of students either strongly agreed or agreed with the statement: I have had experience of the use of translators (either formal or informal).

$64 \%$ of students either strongly agreed or agreed with the statement: I have had experience of dealing with family groups in the dental setting.

$100 \%$ of students either strongly agreed or agreed with the statement: I have had experience of using dental recording and management software.

$78 \%$ of students either strongly agreed or agreed with the statement: I have been able to manage my time effectively.

$78 \%$ of students either strongly agreed or agreed with the statement: I have increased my experience of working as a team.

$100 \%$ of students either strongly agreed or agreed with the statement: The materials that were provided were familiar to me and of good quality.

$86 \%$ of students either strongly agreed or agreed with the statement: I have had the opportunity to work chairside with a dedicated dental nurse.

$64 \%$ of students either strongly agreed or agreed with the statement: I have had experience of working with other health care professionals.

$88 \%$ of students either strongly agreed or agreed with the statement: I have gained experience in instrument decontamination.

$83 \%$ of students either strongly agreed or agreed with the statement: The supervisor was helpful and approachable.

$68 \%$ of students either strongly agreed or agreed with the statement: Feedback and assessment of clinical work was in line with that at the Institute. 
$77 \%$ of students either strongly agreed or agreed with the statement: The whole experience of Outreach has increased my awareness of dentistry outside the hospital setting.

$35 \%$ of students either strongly agreed or agreed with the statement: I have been able to undertake whole patient care.

\section{Patient information}

Patients are advised prior to treatment that they will be treated by a dental student. They sign a patient contract detailing the arrangements for treatment by students and the patient and student commitment to each other. No patient charges are levied in any of our centres; the curriculum content drives patient selection in line with required learning outcomes. The Primary Care Trust partners who share Local Improvement Finance Trust costs with Leeds Dental Institute acknowledge the benefit to local residents but we have specifically avoided any negotiation of a Units of Dental Activity (UDA) equivalent contract as part of student activity.

\section{DISCUSSION}

Dental training outside the usual dental school setting has for many years been regarded as beneficial to undergraduate learning. In $1977^{1}$ Holloway and Dixon noted that for successful clinical teaching there were certain requisites: suitable premises, suitable staff and a clinical course organised in a compatible way. Elkind $^{2}$ notes that in today's health care environment we also need to add compatibility with health policy, the support of stakeholders and funding to our list of requirements.

As an increasing number of UK dental schools are developing Outreach programmes, there are opportunities to learn from each other's experiences and best practice. Each establishment will have developed their programme in different ways and in response to a variety of educational and financial goals. The educational and financial climates prevailing during the planning and running of each site will also have had a bearing on its design and use.

It might be helpful to look at the 'Leeds Experience' in the context of the findings in other dental Outreach establishments and analyse and compare what went well and the challenges we still face.

\section{WHAT WENT WELL}

\section{Facilities}

At Leeds, we were fortunate to be able to have our undergraduate teaching facilities designed and built to our specification, removing a number of difficulties encountered by other authors. In other schemes, ${ }^{3}$ existing facilities in community dental clinics were used. These often consisted of separate surgeries, making staff supervision more difficult. The number of dental chairs available at these sites was not consistent with normal student groupings and staff student ratios. Staff supervising students in enclosed surgeries were only able to supervise four operating students, whereas in our open plan clinic a single staff member was able to supervise our normal group of eight students, improving the cost effectiveness $^{3}$ of this form of teaching.

Our clinic was newly equipped and all equipment and materials were selected by Leeds Dental Institute, to provide a consistent standard of facilities throughout all of our learning environments. Other schemes $^{4}$ were not so fortunate in this regard. Our student feedback indicates that they appreciated the facilities and materials provided. All students gained a working familiarity with patient management systems and image storage and also gained valuable insight in the processes involved in instrument decontamination - a difficult subject to teach in our existing dental hospital environment of centralisation of such procedures. Other authors $^{3}$ have found that cross-infection control has been an issue in some of their Outreach settings.

\section{Patient base}

Dental teachers have been aware for some time that the patients who attend dental school undergraduate clinics are often not representative of the community at large. ${ }^{1}$ Therefore student experience is likely to be enriched by exposure to a more diverse population group during their training, in order to develop appropriate patient management and communication skills. Eaton et al. ${ }^{5}$ and Elkind ${ }^{3}$ reviewed undergraduate Outreach experience internationally and found two of the beneficial effects of Outreach placement were to give exposure to a more diverse, 'real world' patient group and improve communication and interpersonal skills.

More recent data from the $\mathrm{UK}^{2,4}$ confirmed these findings. Our student feedback demonstrated an increased cultural awareness and improved communication skills, learning outcomes that were specifically targeted when designing our Outreach programme. The population attending our Outreach centre comprised in excess of 95\% ethnic minority groups and an estimated 50\% of these patients required some level of interpretation assistance. This allowed our students to develop a range of additional communication skills and an increased level of cultural awareness. These patients also tended to attend in extended family groups which increased student experience of dental care within a family group, ${ }^{5}$ similar to that experienced in many dental practices.

\section{Team-working}

Opportunities for team-working are essential elements within the clinical undergraduate course and well designed Outreach placement can facilitate this..$^{2,5,7}$ When a number of health care professionals are able to train alongside each other, a range of team working opportunities exist. Dental schools may be able to alleviate some of their initial problems by considering this option. Elkind et al. ${ }^{3}$ noted that in their Outreach sites students worked in pairs, acting as operator and nurse. This activity was seen by nurses to actively interfere with teamworking. Our students valued the support from the student dental nurses and chairside support has been reported by other studies to be seen as important.,7,9 Feedback from Leeds students showed an appreciation of the support that the student nurses provided.

Leeds Dental undergraduates have the opportunity to refer patients to the dental hygiene and dental therapy students and receive patients back for further care. Communication with the laboratory takes place in a more 'real life' environment ${ }^{7}$ than is usually the case with a laboratory that is incorporated in the dental school. ${ }^{4}$ Good communications skills can be fostered between undergraduates and dental technology 
students in an inter-professional learning setting. Initially our undergraduate to hygiene therapy student ratio was high, saturating the resources of the hygiene therapists and limiting further referral and interaction. Plans are now in hand to alter this ratio as our number of Outreach sites increases. Other authors ${ }^{7}$ have also found that students enjoy and value opportunities for team-working.

\section{Time management}

Almost $80 \%$ of Leeds students felt that they had improved their time management skills in the Outreach setting, which has reinforced the findings of a number of other authors. ${ }^{2,4}$ Environmental factors such as increased nursing support, electronic patient management systems and patient expectation are likely to have contributed to this.

\section{Staff support}

From the outset we realised that staff training, development and support were going to be key factors in the success of the Leeds Outreach programme. It was acknowledged that isolation may be a problem for Outreach teachers who did not already have a network of contacts within the Dental Institute and other centres within the locality. This was a finding of Elkind et al., ${ }^{3}$ who made suggestions on how this may be overcome. The suggestions by these authors tie in closely with the support that we designed for Leeds Outreach teachers in terms of feeling part of the Institute team, developing a network of support, mentoring, clinical and teacher training and opportunities for wider networking. ${ }^{9}$ The majority of students enjoyed working with our Outreach teacher, found her helpful and approachable and found the level of clinical feedback and assessment consistent with that in the Institute. This is encouraging in terms of the need for consistency and quality ${ }^{3}$ of teaching in the Outreach environment.

Networking and support not only extended to the Outreach teacher. Support via contact with nurse managers and clinical dental nurses at the Institute was also provided for the senior dental nurse employed in the Outreach unit. This was deemed essential for a nurse without experience of providing facilities and support for undergraduates. The combination of a well trained senior nurse together with the presence of dental nurse tutors and hygiene therapy tutors meant that the Outreach teacher was not the only health care professional that the students had to provide information and feedback. This also had the advantage of reducing the isolation and responsibility felt by Outreach teachers in other papers. ${ }^{3}$

For our teachers to have career flexibility and also in order to feel that their teaching skills are developing, opportunities are provided for formal postgraduate training in learning and teaching. Although other authors have not touched on this aspect, it is likely that individuals who have a real interest in helping students to learn in a clinical environment are also keen to develop these skills and expand them into other areas of the learning environment. A career pathway of a series of developmental options for these colleagues will hopefully improve career satisfaction, staff retention, ${ }^{3}$ and their perception of being a valued member of the team.

We were particularly fortunate in being able to recruit a full time teacher as other authors ${ }^{3}$ have found difficulties associated with consistency, training and clinical governance in relation to part time staff.

\section{CHALLENGES}

\section{Patient base}

There were, of course, disadvantages associated with such a specific group of patients. Many adult patients had never experienced any dental care at all or had only attended for extractions when in pain. As in other centres reported, ${ }^{4}$ the level of dental disease was extremely high and oral mucosal pathology, advanced periodontal disease and untreated caries were common. Students had to manage patients' lack of awareness of dental disease, prevention and treatment, as well as dealing with cultural issues surrounding tooth loss and dental treatment.

Despite the high level of support provided by Leeds Dental Institute, our teacher still reported a steep learning curve, $^{3}$ particularly in relation to dealing with patients from this specific area and of managing student learning experience.

\section{Patient attendance}

As with other Outreach schemes, ${ }^{4,8}$ the Leeds Outreach programme had initial difficulties with patient reliability. Patients were reminded of their obligations regarding their 'contract of care' and patients who failed more than one appointment were not offered further care with a student. Patients were also given telephone reminders the day before their appointment. After an initial period of poor attendance, the pattern of attendance settled to relatively good levels.

\section{Clinical experience}

It is important to realise that clinical experience is not only numbers and types of operative treatment, but is in fact the whole experience of patient and team interaction to achieve the desired clinical outcome.

Many of our students felt that they had not carried out 'whole patient care' due to constraints imposed by our patient base. The range of treatments carried out was relatively narrow in the first few months, the main workload comprising basic preventive, restorative and periodontal procedures. Due to our undergraduate/ hygiene therapy ratio it was not possible to refer as much of the basic work as they would have liked. Measures are now in place to improve the referral opportunities and to broaden the range of patients attending the Outreach centre.

Even though our Outreach experience has been carefully planned and integrated within the undergraduate dental curriculum, there has been a constant cycle of learning, reflection and adjustment of our student Outreach experience. In the future, each site, patient base and Outreach team will have its own individual characteristics, which at inception may constitute an unknown factor. This is likely to be true of all new undergraduate Outreach sites. Until there are adequate data to truly support the value of learning experience ${ }^{5}$ in the Outreach setting, this level of monitoring and refinement will be necessary to 
ensure an appropriate learning forum for our students. It is therefore important that the Outreach experience is, for the present, retained within the control of an established learning centre such as a dental school, where a range of learning options is available to address any inequalities or deficiencies in the Outreach experience.

The successful introduction of a model of independent but well supported and managed clinical teaching units as part of Leeds Dental Institute has allowed our dental students to access both modern learning and teaching in a community based locality, while retaining access to the conventional centre of teaching and clinical excellence in a dental school and hospital. As the future recruitment of conventional clinical academic staff becomes a challenge, the combination of satellite clinics with central existing schools can deliver the number and quality of dental graduates a modern health service demands.

1. Holloway P J, Dixon P A. Extra-mural experience for undergraduate dental students. Br Dent J 1977; 143: 146-150.

2. Elkind A. Outreach teaching: is this the future for dental education? Br Dent J 2002; 193: 111-112.

3. Elkind A, Watts C, Qualtrough A et al. The use of Outreach clinics for teaching undergraduate restorative dentistry. Br Dent J 2007; 203: 127-132.

4. Elkind A, Blinkhorn A S, Blinkhorn FA et al. Developing dental education in primary care: the student perspective. Br Dent J 2005; 198: 233-237.

5. Eaton K A. Outreach teaching--coming soon to a practice near you? Primary Dent Care 2005; 12: 115-116.

6. Bartlett D W, Woolford M. Team training at an Outreach dental unit. Eur J Prosthodont Restor Dent 2003; 11: 133-135.

7. Smith M, Lennon M A, Brook A H, Ritucci L, Robinson $P$ G. Student perspectives on their recent dental Outreach placement experiences. Eur J Dent Educ 2006: 10: 80-86.

8. Elkind A, Potter C, Watts $C$ et al. Patients treated by dental students in Outreach: the first year of a pilot project. J Dent Educ 2005; 9: 49-52.

9. Eaton K A, de Vries J, Widstrom E et al. 'Schools without walls?' Developments and challenges in dental Outreach teaching - report of a recent symposium. Eur J Dent Educ 2006; 10: 186-191.

\section{Appendix 1 Student questionnaire on outreach experience at Westbourne Green}

Please tick the box which most closely agrees with your experience at Westbourne green. $S A=$ strongly agree, $A=$ agree, $D K=$ don't know, D = disagree,

$\mathrm{SD}=$ strongly disagree.

\begin{tabular}{|c|c|c|c|c|c|}
\hline & SA & A & DK & D & SD \\
\hline \multicolumn{6}{|l|}{ I had the opportunity to experience a range of cultures. } \\
\hline \multicolumn{6}{|l|}{ I better understand how culture can affect patient dental experience and attitude. } \\
\hline \multicolumn{6}{|l|}{ I have had the opportunity to improve my communications skills. } \\
\hline \multicolumn{6}{|l|}{ I am aware of the skills necessary to communicate with patients for whom English is difficult. } \\
\hline \multicolumn{6}{|l|}{ I have had experience of the use of translators (either formal or informal) } \\
\hline \multicolumn{6}{|l|}{ I have been able to undertake whole patient care. } \\
\hline \multicolumn{6}{|l|}{ I have had experience of dealing with family groups in the dental setting. } \\
\hline \multicolumn{6}{|l|}{ I have had experience of dental recording and management software. } \\
\hline \multicolumn{6}{|l|}{ I have been able to manage my time effectively. } \\
\hline \multicolumn{6}{|l|}{ I have had increased experience of working as a team. } \\
\hline \multicolumn{6}{|l|}{ I have had experience of working with other health care professionals } \\
\hline I have been able to refer patients to other dental care professionals as part of routine dental care. & & & & & \\
\hline I have had opportunities to improve my time management skills. & & & & & \\
\hline
\end{tabular}

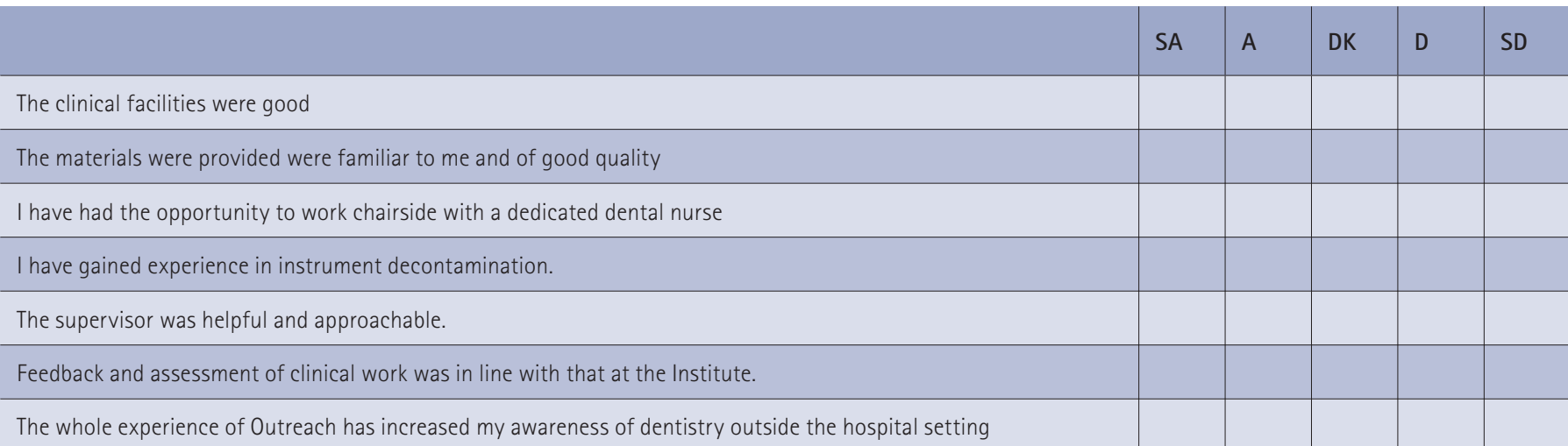

The whole experience of Outreach has increased my awareness of dentistry outside the hospital setting

We would be grateful for any constructive comments that you may have that we may use to help us improve Outreach experience for future groups of students

Thank you for your time and your comments. 